\section{Prevalência de dependência alcoólica em serviços de atenção primária à saúde de Bebedouro, São Paulo, Brasil}

\author{
Prevalence of alcohol addiction among users of \\ primary healthcare services in Bebedouro, \\ São Paulo State, Brazil
}

\section{${ }^{1}$ Escola de Enfermagem, Universidade de São Paulo, São Paulo, Brasil. \\ Correspondência D. Vargas \\ Departamento de Enfermagem Materno- Infantil e Psiquiátrica Escola de Enfermagem, Universidade de São Paulo. Av. Dr. Enéas de Carvalho Aguiar, 419, São Paulo, SP 05403-000, Brasil. vargas@usp.br}

\begin{abstract}
This cross-sectional study aimed to estimate the prevalence of alcohol addiction as detected by primary healthcare services, analyzing its association with clinical, socio-demographic, and behavioral characteristics. The sample included 755 individuals who attended primary healthcare services in the city of Bebedouro, São Paulo State, Brazil, from August 30 to September 30, 2006. Alcohol addiction was investigated using the Alcohol Use Disorders Identification Test (AUDIT). According to the findings, 9.8\% of the sample showed scores suggestive of alcohol addiction (zone IV) on the AUDIT. This group consisted predominantly of male individuals 20 to 39 years of age, white, married, and Catholic. A significant association remained with male gender, smoking, single marital status, lower income, and higher schooling. The strongest predictors of this association were male gender $(O R=5.18)$, smoking $(O R=7.01)$, and university schooling $(O R=2.11)$. Alcohol addiction affects a significant proportion of users of primary healthcare services. In general, male smokers with more schooling and who report some clinical diagnosis were more likely to show an AUDIT score suggestive of alcohol addiction.
\end{abstract}

Alcoholism; Health Services; Primary Health Care
Divane de Vargas 1

Márcia Aparecida Ferreira de Oliveira 1 Eutália C. Araújo 1

\section{Introdução}

Na atualidade, o uso de álcool é caracterizado como um fenômeno social que representa um grave problema à saúde pública mundial, fato que tem despertado a atenção das autoridades médicas e sanitárias em diversos países. Nos Estados Unidos, o problema atinge de $5 \%$ a $10 \%$ das pessoas 1; na América Latina de 3\% a 23\% da população são acometidas pela dependência do álcool 2. No Brasil, um levantamento nacional ${ }^{3}$ evidenciou que $12,3 \%$ das pessoas, com idades entre 12 e 65 anos, são dependentes de álcool.

Esses percentuais devem ser levados em conta, uma vez que o consumo abusivo acarreta inúmeras conseqüências negativas à saúde e à qualidade de vida do indivíduo e da população, contribuindo para o aparecimento de morbidades que causam a morte e limitações funcionais. Estimase que, no Brasil, o uso de álcool seja responsável por mais de $10 \%$ dos problemas totais de saúde 4 , o que parece ser evidenciado não só pela alta prevalência de alcoolistas ocupando leitos de unidades de internação em hospitais gerais do país para tratamento de problemas relacionados direta ou indiretamente ao uso abusivo de álcool 5,6,7, como também pela presença dessa população nos serviços de atenção primária à saúde 8 . Especificamente neste tipo de atenção, estudos internacionais 9,10,11 têm evidenciado alta prevalência de uso nocivo de álcool e dependência alcoólica em pacientes que procuram esses serviços, atin- 
gindo cerca de $50 \%$ entre os homens e $40 \%$ entre as mulheres ${ }^{9}$. Dados de um estudo realizado pela Organização Pan-Americana da Saúde (OPAS) / Organização Mundial da Saúde (OMS) 12 com pacientes da atenção primária à saúde mostraram que a prevalência de dependência do álcool foi de $2,7 \%$, sendo o terceiro transtorno psiquiátrico mais prevalente, superado somente pela depressão e transtorno de ansiedade generalizada. Nos Estados Unidos, $10 \%$ a $36 \%$ dos usuários da atenção primária à saúde fazem uso abusivo de álcool ou são dependentes deste 13

No Brasil, apesar do uso indevido de álcool ser uma das maiores causas de morbidade e mortalidade, poucos estudos foram realizados sobre a prevalência de indivíduos com esse tipo de problema em unidades de atenção primária à saúde; entretanto, os realizados 14,15,16 evidenciaram uma prevalência significativa de pessoas com problemas relacionados ao álcool nesses serviços. Estudo transversal realizado no Sul do país 14 encontrou prevalência de 6,3\% de dependência de álcool entre usuários de serviços de atenção primária à saúde; outro estudo 15 , avaliando os padrões de uso de álcool em usuários desses serviços em dois municípios do interior de Minas Gerais, verificou uma porcentagem de 3,8\% de possível dependência alcoólica. Pesquisa realizada em um município do Nordeste brasileiro 16 apontou que $12,82 \%$ dos sujeitos que procuraram o serviço de atenção primária à saúde faziam uso de álcool de maneira nociva.

De modo geral, o levantamento da literatura brasileira sobre a problemática do álcool e do alcoolismo em serviços de atenção primária à saúde aponta a carência de estudos que possibilitem maiores dados sobre essa condição. Assim, em virtude da grande prevalência do uso abusivo do álcool e dos problemas relacionados a ele e diante da importância dos serviços de atenção primária à saúde, estratégicos para a implementação de ações de identificação precoce e de prevenção, torna-se oportuna a realização de estudos que envolvam regiões ainda não mapeadas, pois, além de determinar a prevalência de possível dependência alcoólica entre usuários de serviços de atenção primária à saúde dessas regiões, os resultados desse tipo estudo permitirão ampliar o conhecimento da temática, além de possibilitar a confirmação e/ou comparação com resultados de estudos já realizados no Brasil.

As estratégias de controle e prevenção do uso do álcool devem ser propostas com base em estudos que indiquem a dimensão do problema existente 17 , já que o resultado de estudos anteriores 14,15,16 têm revelado que uma parcela significativa de usuários de serviços de atenção primária apresenta problemas relacionados ao uso abusivo do álcool. Assim, o objetivo desse estudo foi determinar a prevalência de provável dependência do álcool em serviços de atenção primária à saúde de um município do interior do Estado de São Paulo, Brasil, analisando sua associação com características clínicas, sócio-demográficas e comportamentais.

\section{Metodologia}

Estudo transversal que objetivou verificar a prevalência de dependência alcoólica entre indivíduos que procuram atendimento de saúde nos serviços de atenção primária à saúde de Bebedouro, município com aproximadamente $80 \mathrm{mil}$ habitantes, localizado na região Noroeste do Estado de São Paulo. A rede de serviços de atenção primária à saúde de Bebedouro é composta por duas unidades básicas de saúde (UBS), quatro conglomerados que incluem uma UBS e Programa Saúde da Família (PSF), além de sete unidades de saúde da família.

Os dados foram coletados nos quatro conglomerados que agregam a UBS e unidade de saúde da família (USF). A amostra constituiu-se de 755 sujeitos maiores de 18 anos de ambos os sexos que procuraram atendimento nos locais de coleta, independentemente do motivo. Todos foram recrutados segundo critérios de conveniência.

O trabalho de campo foi realizado entre $30 \mathrm{de}$ agosto e 30 de setembro de 2006. Dez estudantes de enfermagem foram previamente treinados para atuarem como entrevistadores. A coleta ocorreu na recepção de cada serviço, e o preenchimento do instrumento não ultrapassou dez minutos.

O instrumento utilizado para a coleta dos dados foi o Alcohol Use Disorders Identification Test (AUDIT). Trata-se de um screening com reconhecimento mundial, desenvolvido pela OMS na década de 1980, com objetivo de identificar, em serviços de saúde de diferentes níveis e contextos, bebedores de risco. Um dos primeiros testes de rastreamento desenvolvidos especificamente para utilização em serviços de atenção primária à saúde 18, o AUDIT, mede consumo, sintomas de dependência e conseqüências pessoais e sociais do beber. O teste aborda o padrão de consumo e suas conseqüências nos últimos 12 meses, por meio de dez itens, sendo três sobre o uso de álcool, quatro sobre dependência e três sobre problemas decorrentes do consumo 18 . As alternativas de respostas vão de 1 a 4, e as altas pontuações são indicativas de problemas, permitindo classificar o usuário em uma de quatro zonas de risco, de acordo com o escore total obtido: zona I (até 7 pontos: indica uso de baixo risco ou abstinência); 
zona II (de 8 a 15 pontos: indica uso de risco); zona III (de 16 a 19 pontos: sugere uso nocivo) e zona IV (acima de 20 pontos: sugestivo de dependência). Estudo brasileiro utilizando o AUDIT 19 apontou sensibilidade de $87,8 \%$ e especificidade de $81 \%$, sendo o teste considerado satisfatório para estudos em serviços de atenção primária à saúde.

Considerando que escores AUDIT superiores a 20 são sugestivos de dependência alcoólica, para efeito de análise dos dados apresentados, a "dependência do álcool" deve ser interpretada no decorrer desse estudo como "provável dependência do álcool".

Além do AUDIT, os sujeitos responderam a três questões referentes, respectivamente, ao uso de tabaco, à presença de patologias crônicas e/ ou daquelas que poderiam estar relacionadas ao alcoolismo, como úlceras e gastrites.

As variáveis independentes investigadas foram sexo, idade, raça, religião, renda familiar, escolaridade, estado civil, tabagismo e presença de patologias crônicas e ou relacionadas à dependência alcoólica. A análise dos dados foi realizada pelo programa SPSS (SPSS Inc., Chicago, Estados Unidos), por meio do qual foram feitas comparações entre consumo de álcool e prevalência de dependência alcoólica em relação às variáveis sócio-demográficas (sexo, idade, raça, religião, renda familiar, escolaridade, estado civil), comportamentais (tabagismo) e clínicas (patologias associadas). Em um primeiro momento, realizou-se análise logística univariada, no intuito de identificar a associação entre cada uma das variáveis.

Os sujeitos foram classificados, conforme o consumo de álcool, em dois grupos: AUDIT I, grupo de referência, de baixo risco ou abstinência; AUDIT IV, grupo de indivíduos com escore sugestivo de dependência. As variáveis que mostraram associação significativa na análise univariada foram introduzidas, passo a passo, no modelo logístico múltiplo $(\mathrm{p}<0,05)$.

O nível de significância adotado foi de $5 \%$. As estatísticas com p descritivo menor do que ou igual a 0,05 foram consideradas significantes. As significâncias do qui-quadrado do teste de Hosmer-Lemeshow variaram de 0,55 a 0,65 , indicando bom ajuste dos modelos. A presença ou não de multicolinearidade foi testada em cada um destes.

Na regressão logística, a verificação do ajuste do modelo foi realizada por meio dos seguintes testes: qui-quadrado, para mudança do valor -2log verossimilhança $(p<0,05)$, e Hosmer-Lemeshow ( $p>0,10$ ). Empregou-se, ainda, a curva de ROC para especificar o desempenho do AUDIT na classificação dos indivíduos corretamente.
Os procedimentos éticos do estudo são representados pela aprovação do projeto pelo Comitê de Ética em Pesquisa com Seres Humanos das Faculdades Integradas Fafibe e pela assinatura do termo de consentimento livre esclarecido pelos sujeitos que aceitaram participar da pesquisa.

\section{Resultados}

A prevalência de dependência alcoólica detectada pelo AUDIT na população estudada $(n=755)$ foi de 9,8\%. A amostra do estudo constituiu-se predominantemente de sujeitos do sexo feminino, casados, brancos, católicos, com Ensino Fundamental e renda familiar entre um e cinco salários mínimos. A maioria recorreu ao serviço em busca de atendimento na clínica geral (Tabela 1).

A análise estatística dos dados foi realizada com uma subamostra constituída por 666 indivíduos; destes, 592 foram classificados na zona I do AUDIT e constituíram o grupo de referência, enquanto o segundo grupo $(n=74)$ foi classificado na zona IV do AUDIT, portanto com escore sugestivo de dependência alcoólica.

Os sujeitos com escore sugestivo de dependência alcoólica caracterizaram-se, em sua maioria, como do sexo masculino, brancos, casados, católicos, com Ensino Fundamental completo, renda familiar de 1 a 5 salários mínimos, que buscavam atendimento com o clínico geral (Tabela 2). Ao se analisarem as variáveis comportamentais, 54 (73\%) sujeitos classificados na zona IV do AUDIT relataram ser tabagistas.

A associação entre as variáveis estudadas por meio da regressão logística univariada não se demonstrou significativa entre a dependência alcoólica e as variáveis raça, ocupação e especialidade procurada para atendimento na UBS; portanto, estas não foram incorporadas aos modelos múltiplos de regressão logística.

Na regressão logística múltipla, incluíram-se no modelo as variáveis que haviam apresentado significância estatística na análise univariada. Observou-se que as variáveis idade, sexo, escolaridade, renda, estado civil, religião, doenças crônicas e tabagismo estavam positivamente associados à prevalência de dependência alcoólica (Tabela 3).

As variáveis sócio-demográficas, comportamentais e clínicas foram analisadas em conjunto. Os resultados evidenciaram associações significativas entre as variáveis estudadas e a dependência de álcool, exceto para indivíduos com idade menor que vinte anos e os viúvos.

$\mathrm{Na}$ análise pelo modelo múltiplo, mantevese associação significativa entre sexo masculino, 
Distribuição das variáveis incluídas no estudo $(n=755)$. Bebedouro, São Paulo, Brasil, 2008.

\begin{tabular}{|c|c|c|}
\hline Variáveis & $\mathbf{n}$ & $\%$ \\
\hline \multicolumn{3}{|l|}{ Idade (anos) } \\
\hline$<20$ & 52 & 6,9 \\
\hline $20-39$ & 312 & 41,3 \\
\hline $40-59$ & 284 & 37,6 \\
\hline$\geq 60$ & 107 & 14,2 \\
\hline \multicolumn{3}{|l|}{ Sexo } \\
\hline Masculino & 288 & 38,2 \\
\hline Feminino & 467 & 61,8 \\
\hline \multicolumn{3}{|l|}{ Raça } \\
\hline Preta & 111 & 14,7 \\
\hline Branca & 466 & 61,7 \\
\hline Parda & 177 & 23,5 \\
\hline \multicolumn{3}{|l|}{ Estado civil } \\
\hline Solteiro & 155 & 20,6 \\
\hline Casado/Amasiado & 493 & 65,2 \\
\hline Divorciado/Separado & 62 & 8,2 \\
\hline Viúvo & 45 & 6,0 \\
\hline \multicolumn{3}{|l|}{ Escolaridade } \\
\hline Analfabeto & 17 & 2,2 \\
\hline Ensino Fundamental & 476 & 63,0 \\
\hline Ensino Médio & 225 & 29,8 \\
\hline Ensino Superior & 37 & 5,0 \\
\hline \multicolumn{3}{|l|}{ Religião } \\
\hline Católica & 477 & 63,2 \\
\hline Evangélica & 199 & 26,4 \\
\hline Outras & 79 & 10,4 \\
\hline \multicolumn{3}{|l|}{ Ocupação } \\
\hline Desempregado & 31 & 4,1 \\
\hline Cargos operacionais * & 300 & 39,7 \\
\hline Do lar & 218 & 28,9 \\
\hline Aposentado & 70 & 9,2 \\
\hline Motorista & 19 & 2,6 \\
\hline Doméstica & 67 & 8,8 \\
\hline Trabalhador rural & 50 & 6,6 \\
\hline \multicolumn{3}{|c|}{ Renda familiar (salários mínimos) } \\
\hline $1-5$ & 723 & 95,7 \\
\hline$\geq 6$ & 31 & 4,3 \\
\hline \multicolumn{3}{|l|}{ Doenças crônicas } \\
\hline Diabetes & 45 & 6,0 \\
\hline Hipertensão & 156 & 20,6 \\
\hline Gastrite & 58 & 7,7 \\
\hline Úlcera gástrica & 77 & 10,2 \\
\hline Não apresenta & 419 & 55,5 \\
\hline \multicolumn{3}{|l|}{ Especialidade procurada } \\
\hline Clínica geral & 401 & 53,1 \\
\hline Pediatria & 72 & 9,5 \\
\hline Obstetrícia & 5 & 0,7 \\
\hline Odontologia & 58 & 7,7 \\
\hline Ginecologia & 99 & 13,1 \\
\hline Sala de vacina & 27 & 3,6 \\
\hline Outros ** & 93 & 12,3 \\
\hline
\end{tabular}

* Cargos operacionais compreendidos como balconista, frentista, vendedor;

** Curativos, inalações, aplicações de medicamentos, farmácia. 
Prevalência de dependência alcoólica (AUDIT IV) em relação às variáveis do estudo $(n=74)$.

\begin{tabular}{|c|c|c|}
\hline Variáveis & $\mathbf{n}$ & $\%$ \\
\hline \multicolumn{3}{|l|}{ Sexo } \\
\hline Masculino & 53 & 71,6 \\
\hline Feminino & 21 & 28,4 \\
\hline \multicolumn{3}{|l|}{ Idade (anos) } \\
\hline$<20$ & 1 & 1,4 \\
\hline $20-39$ & 33 & 44,6 \\
\hline $40-59$ & 27 & 36,5 \\
\hline$\geq 60$ & 13 & 17,5 \\
\hline \multicolumn{3}{|l|}{ Estado civil } \\
\hline Solteiro & 21 & 28,4 \\
\hline Casado/Amasiado & 41 & 55,4 \\
\hline Divorciado/Separado & 10 & 13,5 \\
\hline Viúvo & 2 & 2,7 \\
\hline \multicolumn{3}{|l|}{ Religião } \\
\hline Católica & 48 & 64,8 \\
\hline Evangélica & 11 & 14,9 \\
\hline Outras & 15 & 20,3 \\
\hline \multicolumn{3}{|l|}{ Escolaridade } \\
\hline Ensino Fundamental & 49 & 66,2 \\
\hline Ensino Médio & 20 & 27,0 \\
\hline Ensino Superior & 5 & 6,8 \\
\hline \multicolumn{3}{|l|}{ Ocupação } \\
\hline Desempregado & 11 & 14,9 \\
\hline Cargos operacionais * & 24 & 32,4 \\
\hline Do lar & 8 & 10,8 \\
\hline Aposentado & 12 & 16,2 \\
\hline Motorista & 8 & 10,8 \\
\hline Doméstica & 4 & 5,4 \\
\hline Trabalhador rural & 7 & 9,5 \\
\hline \multicolumn{3}{|c|}{ Renda familiar (salários mínimos) } \\
\hline Até 1 & 34 & 46,0 \\
\hline $1-5$ & 37 & 50,0 \\
\hline $6-10$ & 3 & 4,0 \\
\hline \multicolumn{3}{|l|}{ Doenças crônicas } \\
\hline Ausente & 27 & 36,5 \\
\hline Diabetes & 7 & 9,5 \\
\hline Hipertensão & 20 & 27,0 \\
\hline Gastrite & 10 & 13,5 \\
\hline Outras & 10 & 13,5 \\
\hline \multicolumn{3}{|l|}{ Especialidade procurada } \\
\hline Clínica geral & 41 & 55,4 \\
\hline Pediatria & 6 & 8,1 \\
\hline Odontologia & 4 & 5,4 \\
\hline Ginecologia & 1 & 1,4 \\
\hline Sala de vacina & 6 & 8,1 \\
\hline Outros ** & 16 & 21,6 \\
\hline
\end{tabular}

AUDIT: Alcohol Use Disorders Identification Test.

* Cargos operacionais compreendidos como balconista, frentista, vendedor;

** Curativos, inalações, aplicações de medicamentos, farmácia. 
Regressão logística, análise da relação entre dependência alcoólica de acordo com as variáveis significativas no estudo. São Paulo, Brasil, 2008

\begin{tabular}{|c|c|c|c|}
\hline Variáveis & OR & IC95\% & Valor de $p$ \\
\hline \multicolumn{4}{|l|}{ Idade (anos) } \\
\hline$<20$ & 0,736 & $0,42-1,70$ & 0,651 \\
\hline $30-49$ & 1,168 & $1,02-1,34$ & 0,028 \\
\hline $40-59$ & 0,753 & $0,67-0,85$ & 0,000 \\
\hline$\geq 60$ & 1,00 & & \\
\hline \multicolumn{4}{|l|}{ Sexo } \\
\hline Masculino & 5,188 & $4,68-5,74$ & 0,000 \\
\hline Feminino & 1,00 & & \\
\hline \multicolumn{4}{|l|}{ Escolaridade } \\
\hline Ensino Fundamental & 1,00 & & \\
\hline Ensino Médio & 1,596 & $1,42-1,78$ & 0,000 \\
\hline Ensino Superior & 2,119 & $1,70-2,64$ & 0,000 \\
\hline \multicolumn{4}{|l|}{ Renda (salários mínimos) } \\
\hline Até 1 & 1,00 & & \\
\hline $1-5$ & 0,701 & $0,63-0,77$ & 0,000 \\
\hline$\geq 6$ & 0,589 & $0,46-0,75$ & 0,000 \\
\hline \multicolumn{4}{|l|}{ Estado civil } \\
\hline Solteiro & 1,00 & & \\
\hline Casado/Amasiado & 0,452 & $0,40-0,50$ & 0,000 \\
\hline Viúvo & 1,061 & $0,90-1,25$ & 0,481 \\
\hline Divorciado/Separado & 0,172 & $0,13-0,23$ & 0,000 \\
\hline \multicolumn{4}{|l|}{ Religião } \\
\hline Católica & 0,620 & $0,55-0,70$ & 0,000 \\
\hline Evangélica & 0,432 & $0,37-0,50$ & 0,000 \\
\hline Outras & 1,00 & & \\
\hline \multicolumn{4}{|c|}{ Presença de doença crônica } \\
\hline Ausente & 1,00 & & \\
\hline Diabetes & 3,094 & $2,62-3,64$ & 0,000 \\
\hline Hipertensão & 1,731 & $1,52-1,96$ & 0,000 \\
\hline Úlcera/Gastrite & 3,246 & $2,79-3,76$ & 0,000 \\
\hline Outras & 1,353 & $1,16-1,57$ & 0,000 \\
\hline \multicolumn{4}{|l|}{ Tabagismo } \\
\hline Sim & 7,021 & $6,37-7,73$ & 0,000 \\
\hline
\end{tabular}

Categorias de referência: sexo feminino, idade $\geq 60$ anos, estado civil solteiro, renda de até 1 salário mínimo, religião "outras", Ensino Fundamental, ausência de patologias.

fumantes, solteiros, menor renda e maior escolaridade com a dependência alcoólica. Os maiores preditores dessa associação foram encontrados no sexo masculino (odds ratio $-\mathrm{OR}=5,18$ ), fumantes $(\mathrm{OR}=7,01)$ e com nível superior $(\mathrm{OR}=$ 2,11).

Em relação aos grupos etários observou-se uma tendência de aumento conforme a relação de idade, no entanto esta constituiu-se como uma associação de fraca intensidade. A faixa etária entre 30 e 49 foi a que apresentou maior associação com a dependência de álcool. No que se refere à presença de doenças crônicas, o diabetes
$(\mathrm{OR}=3,09)$ e úlcera/gastrite $(\mathrm{OR}=3,24)$ configuraram o maior indicativo de associação.

No modelo final, a significância do qui-quadrado do teste Hosmer-Lemershow foi de 0,65, indicando bom ajuste do modelo. O -2log verossimelhança mostrou a contribuição de cada variável no modelo final ajustado, que foi significante $(\mathrm{p}<0,001)$. No modelo logístico final, a área sob a curva foi de 0,82 e a especificidade de 0,70, evidenciando a capacidade do AUDIT para identificar pacientes dependentes com acurácea significativa (Figura 1). 
Figura 1

Área sob a curva ROC entre dependência alcoólica e probabilidade preditiva do modelo final de regressão logística.

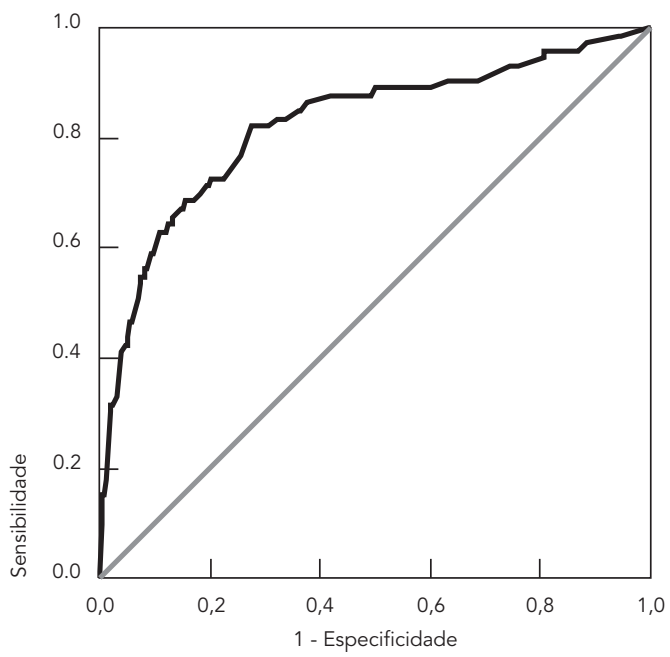

\section{Discussão}

Os estudos transversais são largamente utilizados para investigar a prevalência de condições de saúde, permitindo melhorar o planejamento das ações e gerar hipóteses de novos estudos 20 .

Ao analisar os resultados dos dois últimos levantamentos sobre dependência de álcool no Brasil 3,21, encontrou-se, respectivamente, um percentual de $12,3 \%$ e $11,2 \%$ de dependentes na população geral. O resultado encontrado neste estudo está próximo a esses percentuais, ligeiramente inferior, perfazendo $9,8 \%$ na amostra estudada. Este resultado é consistente com estudo que apontou uma prevalência de dependência de álcool entre $6 \%$ e $10 \%$ na população brasileira, existindo uma variação entre $1 \%$ a $10 \%$ do grupo caracterizado como alcoolistas ou "grandes bebedores" 22. Contudo, quando comparado esse resultado com os obtidos em estudos já realizados nos serviços de atenção primária à saúde 14,15, observa-se que a prevalência encontrada foi significativamente maior.

No que se refere à possível dependência do álcool entre usuários de atenção primária à saúde, os resultados encontrados neste estudo mostraram-se destoantes daqueles verificados em estudos prévios da mesma natureza. As hipóteses para essa discrepância podem estar no fato de que alguns estudos, como de Saalfeld \& Silva 14, foram realizados há mais de uma década, além de terem utilizado instrumento de rastreamento diferente do AUDIT, o que pressupõe que novos estudos possam encontrar maior percentual de indivíduos com esse problema, uma vez que, conforme evidenciam os últimos levantamentos nacionais 3,21 , tem havido aumento de possíveis dependentes do álcool no país na última década. Essa elevação certamente irá repercutir nos percentuais de indivíduos com problemas relacionados ao álcool em serviços de atenção primária à saúde.

Em estudo recente realizado em um município de médio porte da zona da mata mineira 15 , o percentual de possível dependência alcoólica encontrado foi significativamente inferior $(3,8 \%)$, sendo possível supor que as próprias características dos municípios exerçam influência nesses resultados, visto que o cenário dessa pesquisa foi realizado em um município de pequeno porte. É importante considerar que aspectos como suporte social, lazer e cultura local possam exercer influência sobre o modo de beber das pessoas. Ronzani 23 sugeriu que habitantes de municípios de pequeno porte apresentam padrão de uso de álcool mais pesado em virtude das características contextuais supracitadas. Além disso, a avaliação do padrão de uso de álcool entre usuários de municípios de pequeno porte torna-se ainda mais relevante se considerarmos as dificuldades de referenciamento de usuários nocivos ou dependentes para tratamento especializado. Assim, os serviços de atenção primária à saúde tornam-se a principal (ou única) via de acesso aos cuidados em saúde para o uso de álcool, o que poderia justificar o maior percentual de possíveis dependentes de álcool encontrados em Bebedouro.

Por outro lado, o fato de os percentuais de possível dependência de álcool terem sido inferiores àqueles encontrados nos últimos levantamentos nacionais 3,21 pode indicar que muitas pessoas com problemas relacionados à dependência do álcool não procuram os serviços de saúde para atendimento. Resultado consistente com apontamentos de estudo 24 segundo o qual, na América Latina, estima-se que, dos mais de 30 milhões de pessoas passíveis de aplicação dos critérios diagnósticos de transtornos por consumo de álcool, apenas $25 \%$ recebem algum tipo de assistência médica. O fenômeno também é encontrado no Brasil, onde o sistema assistencial tem privilegiado as pessoas mais acometidas pelos problemas relacionados ao álcool nos dispositivos de atenção hospitalar, existindo um longo processo de adoecer, no qual o tempo médio entre a detecção de problemas relacionados ao uso de álcool e a busca efetiva por cuidados pode 
chegar a cinco anos. Evidenciam-se, assim, conseqüências diretas e indiretas da falta de acesso dos usuários a práticas de cunho preventivo ou da ausência de efetividade destas nos serviços de atenção primária à saúde 25 .

Quanto à distribuição dos sujeitos em relação ao sexo, os resultados deste levantamento estão de acordo com a literatura no que se refere à predominância de dependência alcoólica no sexo masculino. Dados que são corroborados pelos resultados dos levantamentos e estudos nacionais que apontam a existência de um predomínio do abuso de bebidas alcoólicas entre os homens $3,14,15,16,20,26,27$. No sexo feminino, a prevalência de dependência, embora menor do que entre os homens, alcançou um percentual significativo $(30,7 \%)$, revelando a proporção de uma mulher para cada dois homens. Esta relação parece ser menor do que a verificada na América Latina 27,28 , onde a razão homem $v s$. mulher alcoolista variava de 5:1 a 14:1, no entanto foi semelhante àquela encontrada no último levantamento feito no país ${ }^{3}$, que apontou a proporção de uma mulher para cada três homens dependentes.

Na distribuição dos dependentes quanto à faixa etária, predominou o intervalo de 30 a 49 anos de idade $(44,6 \%)$, resultado semelhante ao encontrado em outro estudo 26 , quando se compara a distribuição dos sujeitos segundo a faixa etária.

O estudo identificou associação estatística significativa entre nível superior e dependência alcoólica, resultado que é corroborado pelo estudo de Almeida \& Coutinho ${ }^{26}$. Todavia, a maior parte das pesquisas tem revelado uma associação inversa entre prevalência de dependência alcoólica e escolaridade 14,20,28,29. Em adição, os resultados do estudo mostraram que a possível dependência do álcool foi encontrada, em sua maioria, entre aqueles indivíduos com maior renda e menor escolaridade. Apesar de ser esperada associação entre maior renda e maior escolaridade, estes resultados parecem ser consistentes com aqueles de pesquisa 30 que aponta que, embora tenha havido melhora da alfabetização e da escolaridade no Brasil na última década, no que diz respeito à renda per capita dos municípios, não se podem observar as mesmas condições favoráveis apresentadas pela educação no período, sugerindo que a escolaridade tenha uma contribuição muito restrita na geração de renda per capita das cidades.

$\mathrm{Na}$ análise das variáveis clínicas, representadas pelas patologias crônicas, encontrou-se associação estatística significativa entre a dependência de álcool e as patologias diabetes e gastrite. $\mathrm{O}$ resultado foi consistente com a literatura, segundo a qual o uso pesado do álcool aumenta o risco para o diabetes tipo II 31,32. A gastrite configura- se dentre as principais comorbidades clínicas do sistema gastrointestinal associadas ao consumo de bebidas alcoólicas 33 .

A associação tabagismo e alcoolismo foi encontrada em $70 \%$ dos indivíduos com dependência do álcool, dado consistente com os de outros estudos. 3,20,21,28,29.

Com relação ao que motivou a procura de atendimento nas unidades estudadas, $55,4 \%$ dos entrevistados buscaram atendimento na clínica médica. Talvez a procura por essa especialidade justifique-se pelos resultados encontrados no que se refere à associação do alcoolismo a patologias crônicas, como hipertensão, diabetes, cardiopatias e gastrite. Deve-se ressaltar que a hipertensão acomete mais de um quarto dos sujeitos.

A inclusão, na amostra da pesquisa, de indivíduos que procuraram o serviço na clínica pediátrica e sala de vacinas justificou-se pelo fato de serem serviços que, embora não direcionados à população adulta especificamente, como é o caso da pediatria, são freqüentados por acompanhantes. Nessa população, é possível encontrar indivíduos com transtornos relacionados ao uso e abuso de álcool, fato que pode ser constatado neste estudo, quando se verifica que, dos 74 sujeitos com pontuação na zona IV do AUDIT, 16,2\% procuraram os serviços como acompanhantes ou em busca de vacinas. Isso parece comprovar a presença de pessoas com esse problema nas diversas modalidades de atendimento dos serviços disponibilizados na atenção primária à saúde.

Os dados desta pesquisa apontaram a presença de um número considerável de possíveis alcoolistas na demanda que busca atendimento em serviços de atenção primária à saúde. Ademais, os resultados indicaram que os homens fumantes, com maior escolaridade e com diagnóstico de gastrite e diabetes, compõem os grupos com maior risco para a dependência alcoólica e, portanto, mais suscetíveis à morbidade e à mortalidade relacionadas com o alcoolismo.

Apesar de apresentar limitações, como a nãoaleatorização da amostra, pois esta foi realizada segundo critérios de conveniência, este estudo é relevante, diante da escassez de dados nacionais sobre as questões relacionadas ao uso abusivo e dependência de álcool na população que busca atendimento em atenção primária à saúde. Além disso, o conhecimento dessa situação pode ser precursor de estratégias de intervenção no âmbito da atenção primária à saúde, por se tratar indiscutivelmente da principal porta de entrada por procura espontânea de alcoolistas no sistema de saúde. Dessa forma, este fica em posição favorável para a detecção dos casos e intervenções precoces, em face dos problemas ocasionados pelo uso e dependência de álcool. 


\section{Resumo}

Estudo transversal que objetivou estimar a prevalência de dependência alcoólica em serviços de atenção primária à saúde, analisando sua associação com características clínicas, sócio-demográficas e comportamentais. A amostra constituiu-se de 755 sujeitos que procuraram atendimento de saúde em serviços de atenção primária da cidade de Bebedouro, São Paulo, Brasil, entre 30 de agosto a 30 de setembro de 2006. Para a investigação da dependência alcoólica, utilizou-se o Alcohol Use Disorders Identification Test (AUDIT). Os resultados apontaram que 9,8\% da amostra obtiveram, no AUDIT, pontuação sugestiva para dependência alcoólica (zona IV). Esse grupo compôsse predominantemente por indivíduos do sexo masculino, com idade entre 20 e 39 anos, brancos, casados, católicos. Mantiveram associação significativa com a dependência alcoólica sexo masculino, fumantes, solteiros, menor renda e maior escolaridade. Os maiores preditores dessa associação foram encontrados para o sexo masculino $(O R=5,18)$, fumante $(O R=7,01)$ e com nível superior $(O R=2,11)$. A dependência alcoólica acomete significativa proporção dos usuários atendidos em serviços de atenção primária à saúde. De modo geral, os homens tabagistas com maior escolaridade e com relato de algum diagnóstico clínico apresentaram pontuação no AUDIT sugestiva para a dependência alcoólica.

Alcoolismo; Serviços de Saúde; Atenção Primária à Saúde

\section{Referências}

1. Lewis DC. The role of the generalist in the care of substance abusing patient. Med Clin North Am 1997; 81:831-43.

2. Mariategui JS. Investigación epidemiológica del alcoholismo. Rev Assoc Bras Psiquiatr 1985; 26:109-13.

3. Carlini EA, Galduróz JCF, Noto AR, Nappo SA. II Levantamento domiciliar sobre uso de drogas psicotrópicas no Brasil - 2005. São Paulo: Centro Brasileiro de Informações sobre Drogas Psicotrópicas/Departamento de Psicobiologia, Universidade Federal de São Paulo; 2006.

\section{Colaboradores}

D. Vargas participou da concepção do estudo, revisão da literatura, coleta e análise dos dados, redação e revisão do manuscrito. M. A. F. Oliveira colaborou na revisão da literatura, análise dos dados, redação e revisão do manuscrito. E. C. Araújo contribuiu no tratamento estatístico dos dados, discussão dos dados, redação e revisão do manuscrito.
4. Meloni JN, Laranjeira R. Custo social e de saúde do consumo de álcool. Rev Bras Psiquiatr 2004; 26 Suppl 1:Sl7-10.

5. Noto AR, Moura YG, Nappo AS, Galduróz JCF, Carlini EA. Internações por transtornos mentais e de comportamento decorrentes de substâncias psicoativas: um estudo epidemiológico nacional do período de 1988 a 1999. J Bras Psiquiatr 2002; 51:113-21.

6. Masur J, Cunha JM, Zwicker AP, Laranjeira RR, Knobel E, Sustovich DR, Lopes AC. Prevalência de pacientes com indicadores de alcoolismo internados em uma enfermaria de clínica geral: relevância da forma de detecção. Acta Psiquiátr Psicol Am Lat 1980; 26:125-30. 
7. Ribeiro SM, Martins VCR, Ramos MAA. O subdiagnóstico do alcoolismo no hospital geral. HU Rev 1995; 21:15-28.

8. Ronzani TM, Ribeiro MS, Amaral MB, Formigoni MLOS. Implantação de rotinas de rastreamento do uso de risco de álcool e de uma intervenção breve na atenção primária à saúde: dificuldades a serem superadas. Cad Saúde Pública 2005; 21:852-61.

9. Aalto M, Seppa K, Kiianmaa K, Sillanaukee P. Drinking habits and prevalence of heavy drinking among primary health care outpatients and general population. Addiction 1999; 94:1371-9.

10. Lock CA, Kaner E, Lamont S, Bond S. A qualitative study of nurse's and practices regarding brief alcohol intervention in primary health care. J Adv Nurs 2002; 39:333-42.

11. Fleming DT, Wasserheit JN. From epidemiological synergy to public health policy and practice: the contribution of other sexually transmitted diseases to sexual transmission of HIV infection. Sex Transm Infect 1999; 75:3-17.

12. Organização Pan-Americana de Saúde, Organização Mundial da Saúde. Relatório sobre a saúde no mundo 2001: saúde mental: nova concepção, nova esperança. Genebra: Organização Pan-Americana de Saúde, Organização Mundial da Saúde; 2001.

13. Bradley KA, Bush KR, McDonell MB, Malone T, Fihn SD. Screening for problem drinking: comparison of CAGE and AUDIT. J Gen Intern Med 1998; 13:379-88.

14. Saalfeld V, Silva MRA. Prevalência do alcoolismo em cuidado primário à saúde. Pesqui Méd (Porto Alegre) 1993; 27:5-9.

15. Magnabosco MB, Formigoni MLOS, Ronzani TM. Avaliação dos padrões de uso de álcool em usuários de serviços de Atenção Primária à Saúde de Juiz de Fora e Rio Pomba (MG). Rev Bras Epidemiol 2008; 10:637-47.

16. Cordeiro Q, Michelon L, Ribeiro RB, Kamitsuji C, Silveira CM, Andrade LHG. Triagem para a identificação de uso nocivo de álcool na atenção primária à saúde. Rev Assoc Med Bras (1992) 2006; 52:200.

17. Henrique IFS, De Micheli D, Lacerda RB, Lacerda LA, Formigoni MLOS. Validação da versão brasileira do teste de triagem do envolvimento com álcool, cigarro e outras substâncias (ASSIST). Rev Assoc Med Bras (1992) 2004; 50:199-206.

18. Babor TF, De La Fuente JR, Saunders J, Grant M. AUDIT: the alcohol use disorders identification test. Guidelines for use in primary health care. Geneva: World Health Organization; 1992.

19. Mendéz EB. Uma versão brasileira do AUDIT ( $A l$ cohol Use Disorders Identification Test) [Dissertação de Mestrado]. Pelotas: Universidade Federal de Pelotas; 1999.

20. Primo NLN, Stein AT. Prevalência do abuso e da dependência de álcool em Rio Grande (RS): um estudo transversal de base populacional. Rev Psiquiatr Rio Gd Sul 2004; 26:280-6.
21. Carlini EA, Galduróz JCF, Noto AR, Nappo SA. I Levantamento domiciliar sobre uso de drogas no Brasil - 2001. São Paulo: Centro Brasileiro de Informações sobre Drogas Psicotrópicas/Departamento de Psicobiologia da Universidade Federal de São Paulo; 2002.

22. Galduroz JCF, Caetano R. Epidemiologia do uso de álcool no Brasil. Rev Bras Psiquiatr 2004; 26 Suppl 1:Sl3-6.

23. Ronzani TM. Padrão de uso de álcool entre usuários de APS: estudo comparativo. Rev APS 2008; 11:163-71.

24. Kohn R, Levav I, Caldas de Almeida JM, Vicente B, Andrade L, Caraveo-Anduaga JJ, et al. Los trastornos mentales en América Latina y el Caribe: asunto prioritario para la salud pública. Rev Panam Salud Pública 2005; 18:229-40.

25. Secretaria de Atenção à Saúde, Ministério da Saúde. Saúde mental e atenção básica: o vínculo e o diálogo necessários. http://portal.saude.gov.br/ portal/arquivos/pdf/diretrizes.pdf (acessado em 28/Abr/2009).

26. Almeida LM, Coutinho ESF. Prevalência de consumo de bebidas alcoólicas e de alcoolismo em uma região metropolitana do Brasil. Rev Saúde Pública 1993; 27:23-9.

27. Borini P, Silva CO. Aspectos demográficos, epidemiológicos e sociais do alcoolismo: uma análise de alcoolistas internados em hospital psiquiátrico. Rev ABP-APAL 1989; 11:89-96.

28. Almeida LM, Coutinho ESF. O alcoolismo e o hospital geral: estudo de prevalência. J Bras Psiquiatr 1990; 39:27-31.

29. Costa JSD, Silveira MF, Gazalle FK, Oliveira SS, Hallal PC, Menezes AMB, et al. Heavy alcohol consumption and associated factors: a population-based study. Rev Saúde Pública 2004; 38:284-91.

30. Esteves LA. Schooling and income in the Brazilian counties. Social Science Research Network. http://ssrn.com/abstract=897601 (acessado em 18/ Fev/2009).

31. Carlsson S, Hammar N, Grill V, Kaprio J. Alcohol consumption and the incidence of type 2 diabetes: a 20-year follow-up of the Finnish twin cohort study. Diabetes Care 2003; 26:2785-90.

32. Wannamethee SG, Shaper AG, Perry IJ, Alberti KG. Alcohol consumption and the incidence of type II diabetes. J Epidemiol Community Health 2002; 56:542-8.

33. Laranjeira R, Nicastri S, Jerônimo C, Marques AC, Gigliotti A, Campana A, et al. Consenso sobre a síndrome de abstinência do álcool (SAA) e o seu tratamento. Rev Bras Psiquiatr 2000; 22:62-71.

Recebido em 11/Ago/2008

Versão final reapresentada em 04/Mar/2009

Aprovado em 27/Abr/2009 